\title{
Europe : le premier enfant à quel âge ?
}

Dans une Union européenne qui se caractérise par une fécondité « d'hiver démographique ${ }^{1}$, on pourrait penser que l'âge moyen des femmes à la première naissance des différents pays converge et qu'il évolue avec la même intensité. Cela est-il vérifié ?

E

$\mathrm{n}$ réalité, lâge moyen des femmes à la première naissance varie considérablement pour la dernière année disponible, de 26 ans en Bulgarie à 31 ans en Italie, alors que la moyenne pour l'Union européenne est de 29 ans $^{2}$. Pour la Bulgarie, il convient de considérer la part de premiers enfants nés de mères dites adolescentes, c'est-à-dire âgées de moins de 20 ans, notamment au sein de la population rom de ce pays. En Italie, non seulement cette proportion est faible, mais s'y ajoute un faible pourcentage des naissances hors mariage, ce qui repousse l'arrivée de la première naissance au-delà d'un mariage dont lâge a augmenté, dans le contexte d'un pays dont la fécondité demeure faible, la plus faible de l'Union européenne en 2016 (1,34 enfant par femme).

\section{Une singularité orientale}

Lâge moyen des femmes à la première naissance est plus faible, inférieur à 28,5 ans, dans la partie orientale de l'Union européenne ${ }^{3}$ et dans les trois républiques baltes, soit neuf pays allant de la Bulgarie à l'Estonie en passant par la Roumanie la Hongrie, la Slovaquie, la République tchèque et la Pologne

À l'inverse, cet âge est particulièrement élevé, supérieur à 30 ans, dans trois pays méditerranéens, l'Italie, l'Espagne et la Grèce, ainsi qu'au Luxembourg et en Irlande. Dans cet ensemble, c'est plutôt l'Irlande qui détone alors que sa fécondité est relativement élevée.

Entre ces deux ensembles, figurent quatorze pays dont la plupart se situent en Europe occidentale (Autriche, Allemagne, France...) et septentrionale (Suède, Finlande, Royaume-Uni, Danemark). S'ajoutent deux pays de l'exYougoslavie (Slovénie et Croatie), un pays d'Europe méridionale (Portugal) et les deux pays îliens de Méditerranée, Malte et Chypre. Au sein de ces quatorze, on note que la France compte le plus faible âge moyen des femmes à la première naissance $(28,5$ ans) et a pour la même année 2016 la fécondité la moins abaissée $(1,92)$.

1. Dumont, Gérard-François, «La démographie en Europe : en quoi l'étude de l'impact des données démographiques est-elle pertinente? », Fondation Res Publica, $\mathrm{n}^{\circ} 102,24$ octobre 2016.

2. Eurostat, 52/2018, 28 mars 2018.

3. Ceteris paribus, on ne peut ne pas penser à la distinction géographique faites par J. Hajnal distinguant deux zones en Europe, séparées par une ligne imaginaire allant de Trieste à Leningrad (redevenu SaintPetersbourg). A l'ouest de cette ligne, le mariage était rare et tardif, tandis qu'à l'est, il était précoce et fréquent. Le modèle intermédiaire (semitardif) se voyait en Europe centrale. Hajnal, J., European Marriage Patterns in Perspective, pp.101-143, in Population in History, eds. D.V.Glass and D.E.C.Eversley. London, 1965.
L'ÂGE MOYEN DES FEMMES À LA NAISSANCE DU PREMIER ENFANT DANS LES PAYS DE L'UNION EUROPÉEENNE (À 28)
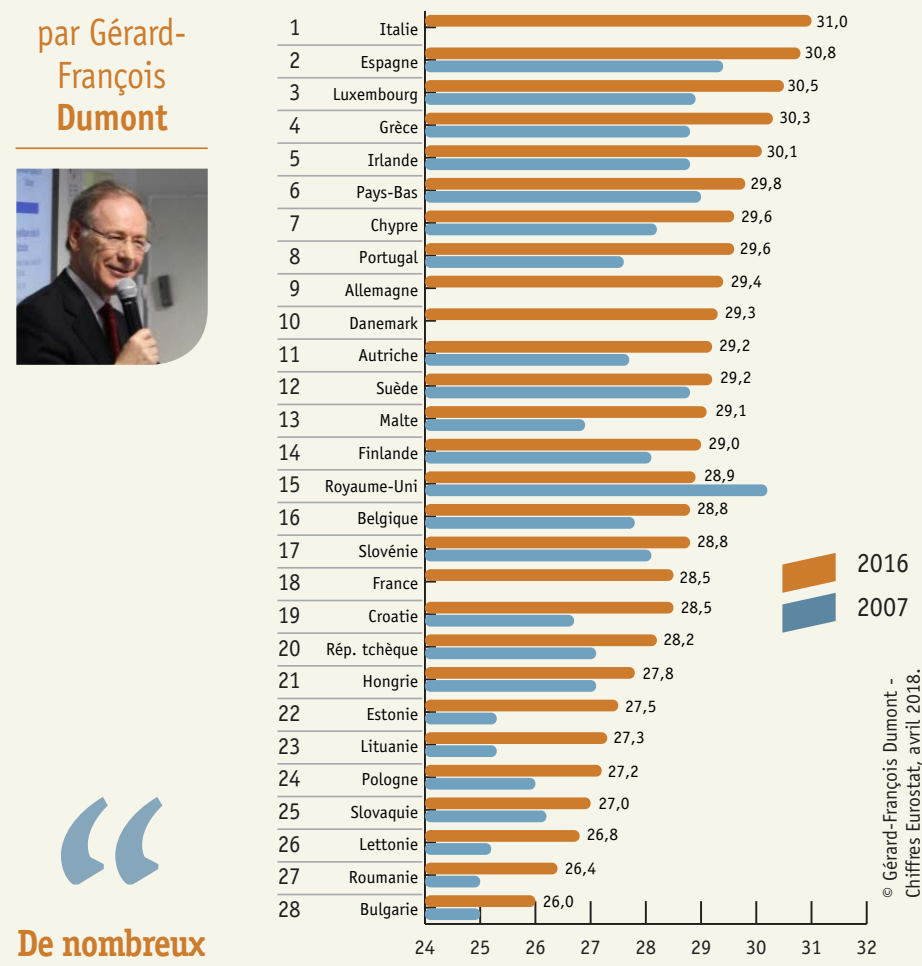

facteurs propres

aux pays doivent

être pris en

considération

pour comprendre

le caractère

fragmenté

des évolutions

démographiques

en Europe.

\section{Le premier enfant plus tard, sauf exception}

La typologie de l'accroissement de l'âge moyen des femmes à la première naissance sur une dizaine d'années (de 2007 à 2016) met en évidence une fragmentation encore plus intense puisqu'il n'y a aucune corrélation entre cet accroissement et l'âge atteint. Les données, pour les pays pour lesquels elles sont disponibles, livrent même un résultat inattendu : la baisse de lâge moyen des femmes à la première naissance au Royaume-Uni.

Quant aux augmentations, leur intensité est fort variable. Elles peuvent atteindre ou dépasser deux ans en Lituanie et Estonie, au Portugal, à Malte ou être très faible, à 0,4 année seulement, en Suède.

Cette diversité de résultats et d'évolution de lâge moyen des femmes à la première naissance signifie que l'appartenance de ces pays à un même ensemble régional et, donc, à de nombreuses réglementations communes que sont les règlements et les directives de l'Union européenne ${ }^{4}$ ne se traduit nullement pas des caractéristiques sociodémographiques semblables. De nombreux facteurs propres aux pays, comme la composition de leur population ou des éléments culturels, doivent être pris en considération pour comprendre le caractère fragmenté des évolutions démographiques en Europe.

4. Dumont, Gérard-François, Verluise Pierre, Géopolitique de l'Europe de l'Atlantique à l'Oural, Paris, PUF, septembre 2016. 